\title{
Double Layered Electrode for Plasmon Antenna
}

\author{
S. Kudoh, J. Kim, K. Nakagawa, and A. Itoh \\ Dep. of Electronics \& Computer Science, College of Sci. \& Tech., Nihon University \\ 7-24-1 Narashinodai, Funabashi, Chiba 274-8501, Japan
}

Recently, for the high recording density technology that aims at recording density of 1 Tbit/inch ${ }^{2}$, heat assisted hybrid recording has been suggested. Near-field optics is one of the important factors for the hybrid recording to shrink an optical spot to a diameter of $50 \mathrm{~nm}$ or below. In this study, we analyzed a double layered plasmon antenna for hybrid recording by Finite Difference Time Domain (FDTD). The double layered plasmon antenna was designed on the resonance wavelength which itself depends on the materials and the shape of the antenna. The results of the calculation showed high power intensity and focused optical spot at the nanosize level.

Key words: heat assisted hybrid recording, near-field optics, double layered plasmon antenna, FDTD.

\section{Introduction}

The current goal in the recording research field is to achieve a recording density of 1 Tbit/inch ${ }^{2}$. For the realization of $1 \mathrm{Tbit} / \mathrm{inch}^{2}$, extremely small magnetic grains are needed, arithmetically 1 bit in the area of 25 $\mathrm{nm} \times 25 \mathrm{~nm}$. However, due to physical limitations, the magnetization of such small grains is easily affected by thermal energy. To overcome this problem in magnetic recording, a high $K_{\mathrm{u}}$ (uniaxial anisotropy energy) material is required ${ }^{1,2}$. However, to record on a high coercive field material, an enormous magnetic recording field is needed. Therefore, to achieve high density, a heat assisted hybrid recording has been suggested ${ }^{1)}$. A hybrid recording system could use intense optical spots to locally heat the medium. Optical spots of $50 \mathrm{~nm}$ or less are required ${ }^{2,3)}$ for a recording density of over 1 Tbit/inch ${ }^{2}$. However, a normal optical system such as a laser can not focus down to this size due to the diffraction limit. Therefore, for heating the small area in hybrid recording, near-field optics has been

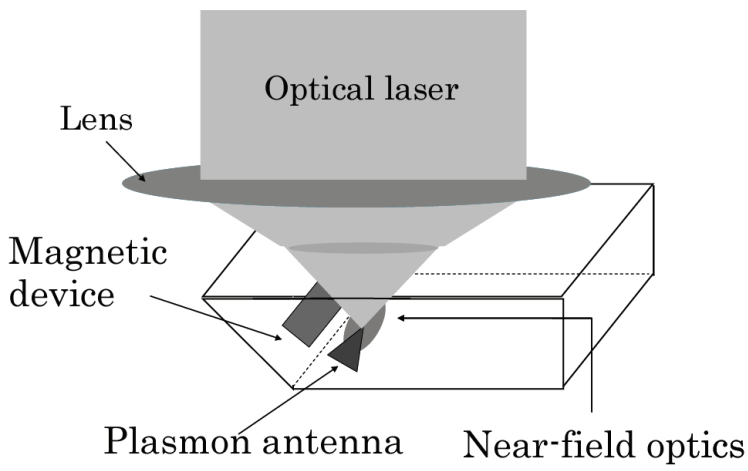

Fig. 1 The schematic diagram of near-field optical advanced hybrid (NOAH) head. The NOAH head mainly consists of a transparent slider, a plasmon antenna and a magnetic device. The slider was made from crystalline glass ${ }^{8}$. introduced.

Near-field optics for hybrid recording has been reported, such as the waveguide type hybrid head ${ }^{4)}$, U patterned hybrid head a surface plasmon and magnetic field applicable synchronously hybridized (SMASH) head $^{5)}$, and bow tie type plasmon antenna ${ }^{6}$. In addition, Matsumoto et al. reported the simulation results that resonance wavelength depends on the shape of the plasmon antenna and its materials ${ }^{7)}$.

To obtain high power intensity in near-field optics, the design of the antenna and its materials is very important. In this paper, we studied the resonance wavelength of a plasmon antenna attributed to the antenna structure and materials specifically for a double layered electrode with Finite Difference Time Domain (FDTD). The model of double layered electrode for plasmon antenna was based on a near-field optical advanced hybrid (NOAH) head, as shown in Fig. ${ }^{8}$.

\section{Simulation with FDTD}

\subsection{Modeling of Plasmon Antenna}

FDTD has worked well for analyzing and optimizing antennas. Using FDTD, the power density $\left.(E)^{2}\right)$ distribution generated by the antenna was calculated. A simulation model of the antenna was designed in order to investigate the influence of material and structure on power intensity, as shown in Fig. 2.The cell size in this simulation is the smallest at $2 \times 2 \times 1 \mathrm{~nm}^{3}$ at the center of the simulation model, near the apex of the antenna. Mur's absorption boundary condition was used in calculating the boundary conditions for this area ${ }^{9}$. Under the variety of the antenna material and the structure, $E^{2}$ distribution was calculated.

The antenna materials in this calculation were $\mathrm{Au}$ and Pt. Relative permeability of the antenna materials was fixed at 1 . The coefficients of the optical properties of each material, such as refractive index and extinction 
coefficient, were changed depending on wavelength, as shown in Table. 110). Antenna length $(L)$ of the longitudinal direction was $100 \mathrm{~nm}$ and $400 \mathrm{~nm}$, the apex radius $(r)$ was $25 \mathrm{~nm}$, the apex angle $(\theta)$ was $40^{\circ}$ and the thickness was $35 \mathrm{~nm}$. The distance from the optical source to the plasmon antenna was $60 \mathrm{~nm}$. The optical spot at diameter $\left(1 / e^{2}\right)$ of Gaussian distribution light, which implies a plane wave multiplied by a Gaussian intensity distribution, was set to be equal to the wavelength of the incident light. The peak intensity of the electric field at the center of the light source was 1 $\mathrm{V} / \mathrm{m}$. The electrical field of polarized light was aligned with the $\mathrm{x}$-axis. The power intensity at a position of 5 $\mathrm{nm}$ from the antenna was observed and plotted, as the lines indicate by the arrows in Fig. 2. The calculation model does not include medium, which can affect the power intensity from the optical properties of the medium, such as refractive index and extinction coefficient. The main interest of this study is about the comparison of two materials, $\mathrm{Au}$ and $\mathrm{Pt}$, on the double layered electrode, and from the distribution of the power intensity at $5 \mathrm{~nm}$ away from the antenna.

\subsection{Resonance Wavelength of Plasmon Antenna}

The calculated results are plotted for the wavelength of the incidence light, as shown in Fig. 3. For the Au antenna, the highest power intensity of $L=100 \mathrm{~nm}$ and $L=400 \mathrm{~nm}$, were at $400(\mathrm{~V} / \mathrm{m})^{2}$ for $\lambda_{\text {res }}=670 \mathrm{~nm}$ and $\lambda_{\text {res }}=1300 \mathrm{~nm}$, respectively. For the Pt antenna, the highest power intensity was at $70(\mathrm{~V} / \mathrm{m})^{2}$ with $L=400 \mathrm{~nm}$. The material of the plasmon antenna had a certain resonance wavelength $\left(\lambda_{\text {res }}\right)$. In addition, all simulation results showed sharp power distributions, such as when the full width half maximum (FWHM) of the $\mathrm{x}$-axis was less than $30 \mathrm{~nm}$, and FWHM of the $\mathrm{y}$-axis was less than $50 \mathrm{~nm}$, as shown in Fig. 4.

In the resonance wavelength, the intensity of the $\mathrm{Au}$ antenna was quite higher than that of the $\mathrm{Pt}$ antenna. As $L$ increased, the resonance wavelength tended to shift to the right in higher wavelength region, as shown in Fig. 3. Optimal incident light by simulation produced a resonance wavelength that showed narrow distribution and high intensity. For example, when the $\mathrm{Au}$ antenna was at $L=100 \mathrm{~nm}$, the FWHM of the $\mathrm{x}$-axis was about $6 \mathrm{~nm}$, as shown in Fig. 4 .

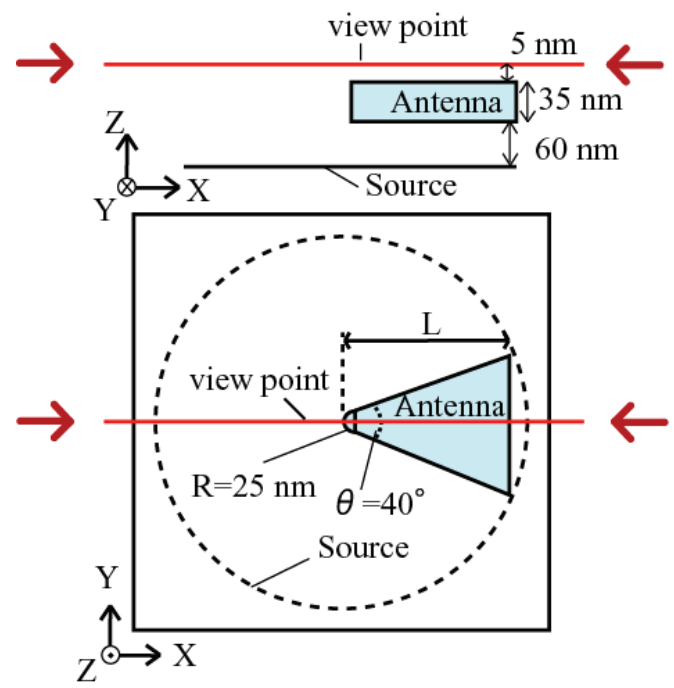

Fig. 2 The schematic diagram of a simulation model for the plasmon antenna. The materials of the antenna were $\mathrm{Au}$ and $\mathrm{Pt}$.

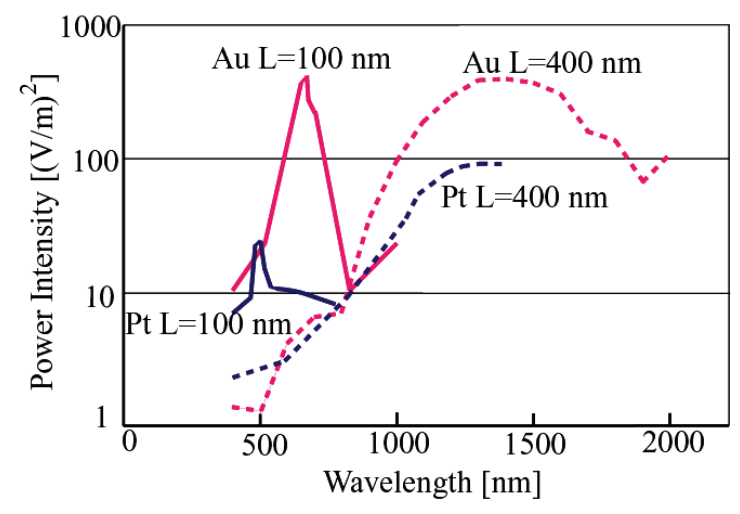

Fig. 3 The intensity measurement shows the peak power intensity as a function of wavelength. Au and $\mathrm{Pt}$ was used as an antenna material. Antenna length $(L)$ was $100 \mathrm{~nm}$ and $400 \mathrm{~nm}$. Solid lines represent the curve of $L=100 \mathrm{~nm}$, dashed lines represent the curve of $L=400 \mathrm{~nm}$.

Table 1 Optical properties of antenna materials ${ }^{10)}$

\begin{tabular}{|c|c|c|c|c|}
\hline \multirow{2}{*}{$\begin{array}{l}\text { Wavelength } \\
\text { [nm] }\end{array}$} & \multicolumn{2}{|c|}{$\mathrm{Au}$ (Gold) } & \multicolumn{2}{|c|}{ Pt (Platinum) } \\
\hline & $\begin{array}{c}\text { Reflective } \\
\text { Index } \\
n\end{array}$ & $\begin{array}{c}\text { Extinction } \\
\text { Coefficient } \\
k\end{array}$ & $\begin{array}{c}\text { Reflective } \\
\text { Index } \\
n \\
\end{array}$ & $\begin{array}{c}\text { Extinction } \\
\text { Coefficient } \\
k \\
\end{array}$ \\
\hline 400 & 1.685 & 1.956 & 1.72 & 3.01 \\
\hline 496 & 0.91 & 1.98 & 1.96 & 3.42 \\
\hline 670 & 0.163 & 3.475 & 2.44 & 4.3 \\
\hline 820 & 0.187 & 5.34 & 2.91 & 5.07 \\
\hline 1409 & 0.473 & 8.96 & 4.91 & 6.95 \\
\hline
\end{tabular}




\subsection{Double Layered Electrode for Plasmon Antenna}

In the double layered electrode, the material design is a key factor. $\mathrm{Au}$ and $\mathrm{Ag}$ for the plasmon antenna showed a high intensity for generating near field optics ${ }^{7)}$. However, when these materials are used as a plasmon antenna in the NOAH head as shown in Fig.1, their durability becomes a problem, such as in the adhesion between $\mathrm{Au}$ and glass, and in the oxidation of Ag. The use of intermediate materials or protective materials can solve the problem. However, the effects on near-field optics have to be considered.

Fig. 5 shows the simulation model to investigate the effects on the underlayer. The structure of the plasmon antenna is a double layered electrode, and consists of a Pt layer of $5 \mathrm{~nm}$ thick and an Au layer of 30 $\mathrm{nm}$ thick. The thickness of the entire double layered electrode was set at $35 \mathrm{~nm}$ for comparison with the single material antenna. The power intensity of the double layered antenna is plotted in Fig. 6. Resonance

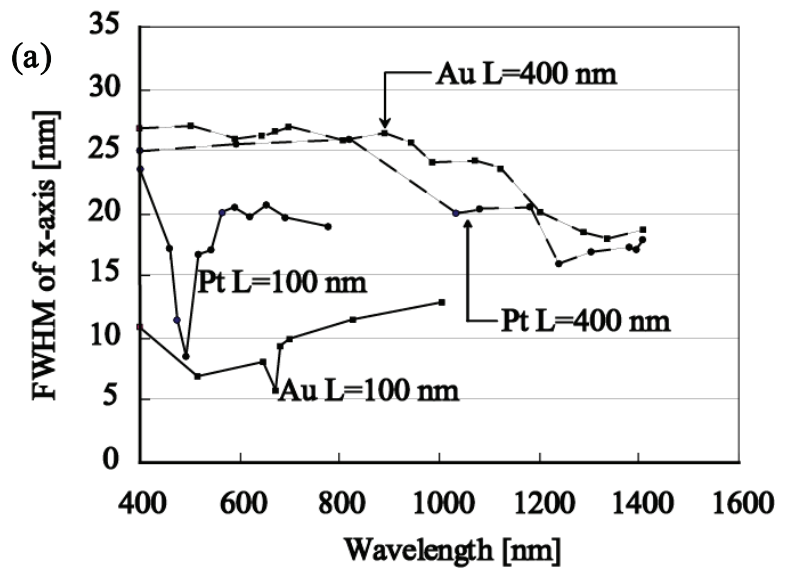

(b)

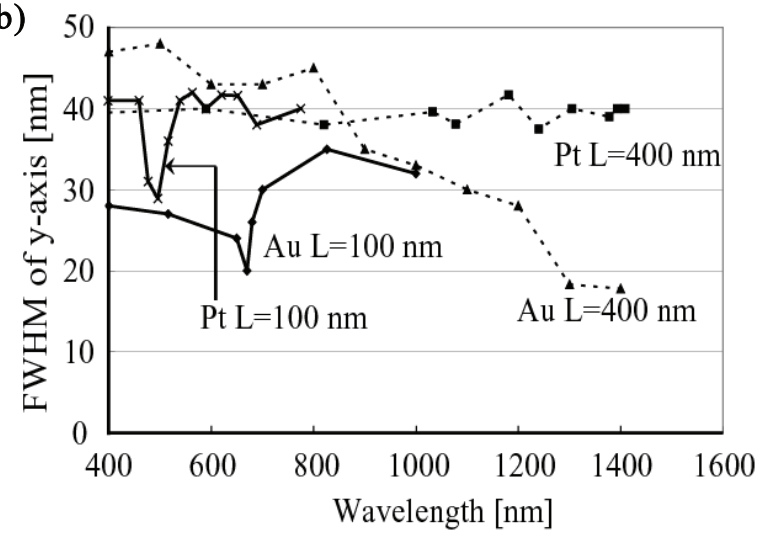

Fig. 4 (a)FWHM of $x^{-}$axis and (b)FWHM of $y^{-a x i s ~ a s ~ a ~}$ function of wavelength. Au and Pt was used as an antenna material. Antenna length $(L)$ was 100 $\mathrm{nm}$ and $400 \mathrm{~nm}$. Solid lines represent the curve of $L=100 \mathrm{~nm}$, dashed lines represent the curve of $L=400 \mathrm{~nm}$. wavelength appeared in the calculated results of the double layered electrode. When $L=100 \mathrm{~nm}$, the resonance wavelength $\lambda_{\text {res }}$ of the double layered antenna was $652 \mathrm{~nm}$, lower than that of the Au antenna $\left(\lambda_{\text {res }}=670 \mathrm{~nm}\right.$, in Fig. 3), and higher than that of the Pt antenna $\left(\lambda_{\mathrm{res}}=496 \mathrm{~nm}\right)$. When $L=100 \mathrm{~nm}$, the intensity of the double layered antenna was about $400 \%$ higher than the Pt antenna. Additionally, in the case of $L=400$ $\mathrm{nm}$, the intensity was about $300 \%$ higher than the Pt antenna. The highest value of power intensity in the double layered antenna did not reach that of the $\mathrm{Au}$ antenna; however, the power distribution of the double layered antenna was concentrated as much as with the result of the Au antenna. In the additional calculated

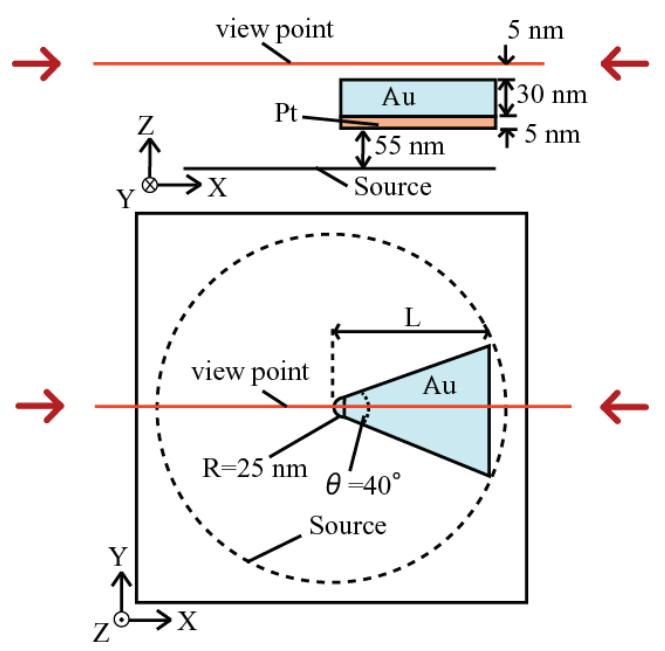

Fig. 5 The schematic diagram of the simulation model for the double layered electrode. Antenna consists of $\mathrm{Au}$ at $30 \mathrm{~nm}$ thickness and $\mathrm{Pt}$ at $5 \mathrm{~nm}$ thickness.

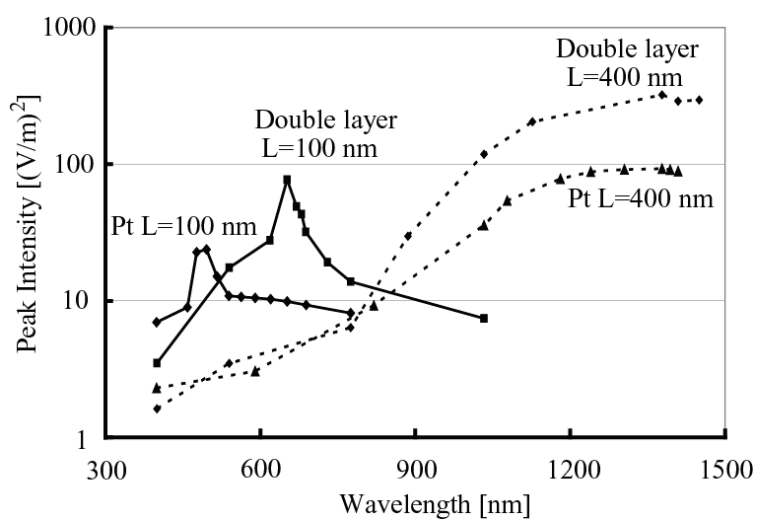

Fig. 6 Power intensity of double layered electrode. The intensity to the $\mathrm{x}$-axis is plotted as a function of wavelength. Solid lines represent the curve of $L=100 \mathrm{~nm}$, and dashed lines represent the curve of $L=400 \mathrm{~nm}$. 
result of the double layered antenna with both $L$ sizes, the FWHM of the $\mathrm{x}^{-}$axis was less than $30 \mathrm{~nm}$, and the FWHM of the y-axis was less than $50 \mathrm{~nm}$, especially where the FWHM was at $7 \mathrm{~nm}$ with $L=100 \mathrm{~nm}$ at $\lambda_{\text {res }}=652 \mathrm{~nm}$ using the double layered antenna.

For hybrid recording, it is quite important to increase the optical efficiency in near-field optics. The reason for using a transport slide in the NOAH head was also for a high efficiency. To estimate optical efficiency $(\eta)$ in this study, the following equation was used

$$
\eta=\frac{\int_{\mathrm{S}} p_{\mathrm{NFO}} \mathrm{dS}}{\int_{\mathrm{S}} \mathrm{p}_{\mathrm{IL}} \mathrm{dS}}
$$

where $p$ NFO is the power intensity distribution of the near-field optics, and $p \mathrm{~L}$ is that of the incident light without plasmon antenna. The optical spot of Gaussian distribution light was set at a diameter of $780 \mathrm{~nm} . S$ is the integrated area under the distribution curve of the calculated power intensity in the light source of diameter $1560 \mathrm{~nm}$, measuring twice the diameter of the spot size. In this configuration, the optical efficiency was decided by the proportion of $S$ from the total area of the power intensity distribution. The optical efficiency of about $87 \%$ for total energy was estimated by FDTD. Therefore, using a resonance wavelength in hybrid recording should obtain a high efficiency.

By using the $\mathrm{Pt}$ underlayer, the resonance wavelength of $\mathrm{Au}$ showed an inclination to shift to that of Pt without changing the property in the resonance wavelength. This result revealed that the design of composite materials, such as the double layered plasmon antenna, could provide a shifting resonance wavelength generated by the incident light.

\section{Conclusion}

According to FDTD, a resonance wavelength depends on the material and structure of the plasmon antenna. The power distribution in the resonance wavelength was highly concentrated with a high intensity. A double layered antenna also had a resonance wavelength between those of the two materials used. The measured results of the double layer antenna, composed of an $\mathrm{Au}$ layer with a $\mathrm{Pt}$ underlayer, showed a high intensity in resonance wavelength with a highly concentrated distribution.

Finally, a narrow distribution and a high intensity of the light of resonance wavelength can be expected to increase the optical efficiency for hybrid recording.

Acknowledgements This work is partially supported by a Grant-in-Aid for Scientific Research of the Ministry of Education, Culture, Sports, Science and Technology in Japan No. 18560348.

\section{References}

1) J. J. M. Ruigrok, R. Coehoorn, S. R. Cumpson and H. W. Kesteren: J. Appl. Phys. 87, 5398 (2000).

2) C. T. Lie, P. C. Kuo, and C. L. Shen: J. Appl. Phys. 94, 2538 (2003).

3) A.V. Itagi, D. D. Stancil, J. A. Bain and T. E. Schlesinger: Appl. Phys. Lett. 83, 4474 (2003).

4) T. E. Schlesinger, T. Rausch, A. Itagi, J. Zhu, J. A. Bain and D. D. Stancil: J. Appl. Phys. 41, 1821 (2002).

5) S. Miyanishi, N. Iketani, K. Takayama, K. Innami, I. Suzuki, T. Kitazawa, Y. Ogimoto, Y. Murakami, K. Kojima and A. Takahashi: IEEE Trans. Magn. 41, 2817 (2005).

6) T. Matsumoto, T. Shimano, and S. Hosaka: Technical Digest of 6th Int. Conf. Near-field Optics and Related Techniques, the Netherlands, Aug. 27-31, 55 (2000).

7) T. Matsumoto, T. Shimano, H. Saga, H. Sukeda: J. Appl. Phys. 95, 3901 (2004).

8) K. Nakagawa, J. Kim, and A. Itoh: J. Appl Phys, 99, 08F902 (2006).

9) G. Mur: IEEE Trans. Microwave theory and techniques, MIT-29, 1073-1077 (1981).

10) Edword D. palik: Handbook of Optical Constants of solids, Academic Press, I - III (1998).

Received May. 31, 2006; Revised July. 10, 2006;

Accepted Aug. 03, 2006 